\title{
émulations
}

\section{Retour critique sur le modèle de ségrégation urbaine de Schelling}

\author{
Michel Forsé, Maxime Parodi
}

Émulations - Revue de sciences sociales

2019, $n^{\circ}$ 31, «Thomas C. Schelling dans les sciences sociales ».

\section{Article disponible à l'adresse suivante}

https://ojs.uclouvain.be/index.php/emulations/article/view/forse_parodi_schelling

\section{Pour citer cet article}

Michel Forsé, Maxime Parodi, « Retour critique sur le modèle de ségrégation urbaine de Schelling », Émulations, n³1, Mise en ligne le 15 novembre 2019.

DOI : 10.14428/emulations.031.07

Distribution électronique : Université catholique de Louvain (Belgique) : ojs.uclouvain.be

(C) Cet article est mis à disposition selon les termes de la Licence Creative Commons Attribution, Pas d'Utilisation Commerciale 4.0 International. http://creativecommons.org/licenses/by-nc/4.0/

Éditeur : Émulations - Revue de sciences sociales / Presses universitaires de Louvain https://ojs.uclouvain.be/index.php/emulations

ISSN électronique : 1784-5734

PUL PRESSES UNIVERSITAIRES 


\title{
Retour critique sur le modèle de ségrégation urbaine de Schelling
}

\author{
Michel Forsé1, Maxime Parodi²
}

\begin{abstract}
[Résumé] Dans les années 1970, Thomas C. Schelling a proposé un modèle pour expliquer le lien entre ségrégation spatiale et préférences individuelles concernant cette ségrégation. Au moyen de simulations, il cherche à montrer qu'un haut niveau de ségrégation globale peut être le résultat collectif de décisions individuelles qui sont loin de viser une telle ségrégation. Cet article montre cependant que les contraintes structurelles du modèle expliquent intégralement les niveaux de ségrégation atteints. Une faible exigence individuelle pour s'entourer de voisins identiques conduit à une ségrégation collective faible et une exigence forte conduit à une ségrégation forte. Les niveaux de ségrégation correspondant à un seuil donné de satisfaction individuelle n'ont rien de surprenant au regard des lois du hasard et de ce que chaque individu souhaite réellement. Le modèle de Schelling ne permet pas de conclure que, de manière générale, de larges ghettos naissent d'innocentes décisions. Ses caractéristiques sont trop particulières pour pouvoir en tirer des enseignements généraux. S'il faut expliquer la formation des "ghettos urbains », il vaut mieux se tourner vers des causes sociales, économiques ou culturelles.
\end{abstract}

Mots clés : ségrégation spatiale, simulations, entropie, modèle dynamique.

\section{A Critical Review of Schelling's Urban Segregation Model}

[Abstract] In the 1970s, Thomas C. Schelling proposed a model which claimed to show that a high degree of spatial segregation can result from individual preferences which do not in themselves aim to achieve such a degree of collective segregation. However, the present paper demonstrates that this model contains several structural constraints which fully explain the obtained levels of segregation. A weak individual demand for similar neighbours leads to low social segregation and a strong demand leads to high segregation. The levels of segregation that are globally achieved for a given threshold of individual satisfaction are not at all surprising in relation to the laws of chance and to what each individual actually wishes. The Schelling model does not allow to conclude that, in general, large ghettos are resulting from innocent decisions. Its characteristics are too specific to draw general lessons. If the formation of "urban ghettos" has to be explained, it is better to investigate social, economic or cultural causes.

Keywords: spatial segregation, agent-based simulation, entropy, dynamic model.

\section{Introduction}

Dans les années 1970, Thomas C. Schelling $(1971$; 1978) a proposé un modèle pour montrer qu'une forte ségrégation spatiale peut être le résultat collectif de préférences individuelles qui ne visent pas, elles, à une telle ségrégation. Même si l'on peut songer à nombreuses applications (Ruoff, Schneider, 2006), c'est incontestablement celle qui a porté sur le cas urbain qui a fait sa notoriété. Elle conduirait à identifier un « effet

\footnotetext{
${ }^{1}$ CNRS, Centre Maurice Halbwachs, France.

2 Sciences Po, OFCE, France.
} 
pervers », ou effet de système : même si des individus sont plutôt tolérants pour ce qui concerne la composition de leur voisinage, et indépendamment d'autres paramètres comme les prix des loyers, les niveaux de revenu, etc., on aboutirait de toute façon à une ségrégation élevée au bout d'un certain temps.

Dans la présentation originale de son modèle, Schelling (1971) est parfaitement conscient que la dynamique qu'il expose - et qui porte sur la mobilité résidentielle au sein d'un quartier - n'est pas la seule explication de ce qui s'observe. Il prend ainsi bien soin de mentionner l'existence au sein de son modèle de contraintes structurelles, mais il reste cependant convaincu que la ségrégation spatiale découle d'abord d'un mécanisme souterrain qu'il compare à une sorte de « main invisible » (le terme exact qu'il emploie est " unseen hand »). Il souligne également, à juste titre, que si tout le monde veut que son groupe soit majoritaire dans son voisinage alors, globalement, la seule solution possible est la ségrégation complète. Plus généralement, il n'oublie pas que certaines exigences en matière de voisins admissibles peuvent conduire à des contraintes de structure qui, en pesant sur les déménagements ou emménagements, finissent par rendre la mixité peu probable voire impossible. Toutefois, ces contraintes structurelles statiques peuvent selon lui être négligées et elles ne sont pas son centre d’intérêt : « La mathématique simple des ratios et des mélanges nous dit quelque chose sur les résultats qui sont logiquement possibles, mais nous dit peu sur le comportement qui conduit ou qui éloigne de résultats particuliers³.» (Schelling, 1971 :147)

Même s'il a proposé plusieurs variantes de son modèle, celle spatiale et bidimensionnelle présentée en 1978 demeure la plus célèbre. Or, il faut reconnaître que si l'on procède aux mêmes simulations que celles de Schelling, on retrouve indéniablement le résultat qu'il met en avant. En l'examinant de plus près, comme nous le ferons, il sera cependant possible de constater que l'interprétation qui en est donnée se trouve faussée précisément par l'absence de prise en compte des aspects structurels. En réalité, leur poids effectif dans l'explication finale de la ségrégation n’a jamais été évalué. Le fait que les préférences individuelles retenues rendent la mixité improbable ou quasi impossible quelle que soit la dynamique aurait pourtant dû conduire à s'interroger très directement sur la portée du modèle.

Ce n'est toutefois pas la seule interrogation. Schelling considère en effet que la seule explication possible des résultats de son modèle est qu'il s'agit d'une simple évolution cumulative. Il ne dit donc pas seulement que des actions individuelles peuvent produire par agrégation un effet collectif non voulu. Il soutient en outre que l'effet non voulu est un supplément de ségrégation. Une telle conclusion aurait dû à nouveau faire douter puisqu'elle revient à remettre en cause ce que l'on sait des lois du hasard et notamment du principe d'entropie $e^{4}$. Elle conduit en effet à penser que de l'ordre (ou, ce

\footnotetext{
${ }^{3}$ Traduction en français des auteurs.

${ }^{4}$ De manière simplifiée, on peut définir ce principe de la manière suivante. Dès lors que seul le hasard intervient, la dynamique d'un système fermé conduit beaucoup plus probablement à l'augmentation du désordre qui y règne. L'ordre se définit par le fait que les éléments de nature différente qui composent un système ne soient pas mélangés. Le désordre correspond
} 
qui revient au même ici, de la ségrégation) est produit spontanément en sus des efforts conscients pour construire un peu d'ordre. Certes, les actions intentionnelles créent de l'ordre. Le problème est de savoir si, au-delà, les conséquences non intentionnelles créent en moyenne plus d'ordre que de désordre. Il y aurait alors bien une sorte d'effet système. Or, d'une manière générale, la pente la plus probable (au regard du principe d'entropie) est au contraire celle qui conduit spontanément au désordre ou ici à une mixité sociale. Se pose alors une nouvelle question : quelle est la généralité du modèle de Schelling ? Car on ne peut se contenter de dire qu'il y a en général une dynamique qui fabrique un peu plus de ségrégation que ce que veulent les individus. Il doit nécessairement y avoir des hypothèses implicites qui expliquent ce devenir. Autrement dit, relativement au modèle général qui stipule que l'effet non voulu le plus probable est le désordre ou la mixité sociale, il faut se demander quelles sont ces hypothèses ou contraintes implicites qui expliquent forcément l'étrange " paradoxe » de Schelling.

Pour répondre à ces interrogations, après avoir rappelé comment se présente le modèle, nous examinerons les niveaux de ségrégation spatiale résultant du seul hasard. Bien qu'il n'ait jamais été produit, cet étalonnage est cependant tout à fait indispensable pour situer un niveau donné de ségrégation. Il permettra de pointer les éventuels décalages dus au processus mis en œuvre par Schelling et mettra ainsi en évidence le rôle des contraintes structurelles. Puis, nous reproduirons les simulations de Schelling en scrutant plus précisément le jeu d'hypothèses à partir desquelles son modèle est formulé. Il sera alors possible de comprendre comment elles construisent de toute pièce les niveaux de ségrégation atteints. Au bout du compte, il apparaîtra que la ségrégation globale est liée à l'intolérance individuelle mais qu'on ne peut pas affirmer qu'une faible intolérance individuelle conduise par effet pervers d'agrégation à une forte ségrégation globale. Cet exposé des présupposés du modèle de Schelling nous permettra au final d'élargir la discussion pour livrer quelques éléments de réflexion sur ce que devrait être un bon modèle en sciences sociales.

\section{Présentation du modèle de Schelling}

Rappelons tout d'abord comment se présente ce modèle dans la dernière version qu'en donne Schelling en 1978. Prenez un échiquier (une matrice $8 \times 8$ ) et placez-y des pions de deux genres différents. Vous avez là une représentation très stylisée d'une aire urbaine où se côtoient deux populations. Ce pourrait être des catholiques et des protestants, des riches et des pauvres ou tout autre clivage. Pour représenter un tel clivage, disons que nous allons répartir des pions noirs et blancs sur l'échiquier. Au départ, ces pions sont rangés alternativement pour représenter l'intégration la plus parfaite possible entre deux communautés.

Schelling ajoute ensuite une règle de déplacement des pions : bien que tolérants, les individus déménageront si, parmi leurs voisins immédiats, un tiers ou moins sont de

à leur mélange. La thermodynamique statistique ou la théorie mathématique de l'information permettent de calculer précisément le degré de désordre ou d'entropie d'un système. 
même couleur qu'eux. En sens inverse, ils seront satisfaits et ne déménageront pas si, parmi leurs voisins immédiats, strictement plus d'un tiers est de couleur identique. Cette règle du tiers peut sembler assez souple. On ne peut pas dire que les individus recherchent la ségrégation; ce n'est qu'à partir du moment où chacun souhaite être majoritaire dans son voisinage que la ségrégation est véritablement visée. En deçà, certaines minorités peuvent s'accepter et le mélange des deux communautés reste possible.

Dans la situation de départ alterné (en enlevant les pions situés aux quatre coins de l'échiquier), tous les individus sont satisfaits de leur voisinage. Schelling effectue alors une petite perturbation. Il commence par retirer au hasard 10 pions blancs et 10 pions noirs, puis il remet 5 pions au hasard, la couleur de chacun étant choisie aléatoirement. Il y a donc finalement 45 pions et 19 cases vides. Cette perturbation suffit le plus souvent à engendrer un peu d'insatisfaction et les pions concernés seront alors déplacés vers des cases vides jusqu'à ce que l'on atteigne un nouvel équilibre où tous seront satisfaits au regard de la règle du tiers. Dans ces conditions, le résultat est surprenant : des quartiers noirs et des quartiers blancs apparaissent sans que personne ne le veuille réellement - du moins, à ce qu'il semble. Comme l'illustre la figure $\mathrm{n}^{\circ} 1$, il y a au final, après tout ce processus de migration, une forte ségrégation. Or, ce n'est pas un résultat marginal. Comme le dit Schelling, réessayez plusieurs fois de jouer à ce jeu et vous aboutirez le plus souvent à une configuration de ce type. En fait, si l'on procède à des milliers de simulations, autour de la ségrégation moyenne constatée, il y a un écart-type qui n'est pas négligeable. Mais la ségrégation moyenne correspond bien au résultat mis en évidence par Schelling.

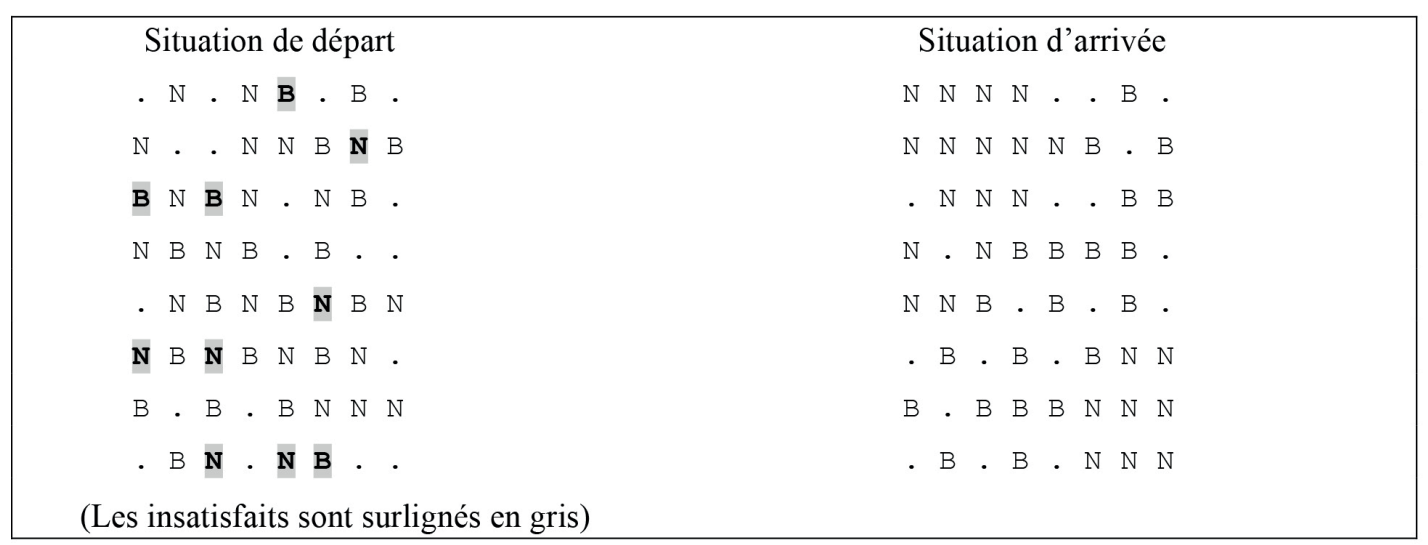

Figure N 1 : Un EXEMPLE d'ÉVOLUTION VERS LA SÉGRÉGATION. SOURCE : SIMULATION DES AUTEURS.

Ce qui est particulièrement frappant dans ce modèle, c'est que tout le monde sait bien que l'ordre n'apparaît pas spontanément. Prenez l'échiquier, mettez-y tous les pions blancs d'un côté, tous les noirs de l'autre et agitez l'échiquier ; s'ils ne sont pas mélangés à la fin c'est un miracle. Dans un système comme celui de Schelling le plus probable est le désordre, c'est-à-dire une certaine intégration des deux communautés. C'est cette 
pente vers le plus probable que le modèle de Schelling semble défier et dès lors, avant de conclure à un paradoxe, il faut se demander si son résultat n'est pas la traduction d'un jeu d'hypothèses implicites qui fait paraître surprenant ce qui n'est que logique.

\section{Un modèle où le mélange devient rapidement impossible}

La première question qui se pose est celle de savoir quelle est la ségrégation attendue pour un seuil de tolérance individuelle donné. À l'évidence, plus la tolérance diminue, moins il y a de configurations possibles sur l'échiquier et plus les situations à forte ségrégation deviennent probables. Mais jusqu'à quel point cela est-il vrai, indépendamment de la dynamique?

Pour le déterminer, il faut commencer par se donner un indicateur du degré de ségrégation d'une distribution sur l'échiquier. L'entropie, telle que définie par Claude E. Shannon (1948) en théorie de l'information (en suivant bien sûr la définition qu'en donne Ludwig Boltzmann en thermodynamique), mesure de manière pertinente et fiable (Kapur, Kesavan, 1992) le degré de désordre d'un système. Notre indicateur d'entropie (celui présenté ci-dessous au tableau $n^{\circ} 1$ pour mesurer la ségrégation) est calibré pour aller de 0 (mélange parfait) à 1 (ségrégation parfaite).

Ensuite, pour mieux comprendre ces aspects structurels ou, disons, statiques, nous avons effectué des millions de tirages aléatoires de pions noirs et blancs sur un échiquier et examiné à chaque fois quel était le seuil de tolérance du pion le moins satisfait de son voisinage. Ceci nous a permis de produire une statistique sur les chances d'obtenir par le seul effet du hasard un échiquier où tous les individus sont satisfaits de leur voisinage pour un seuil donné.

Le grand enseignement de ces simulations est qu'il est quasiment impossible d'obtenir par hasard un échiquier respectant un seuil de satisfaction strictement supérieur à 1/3 (c'est-à-dire le seuil choisi par Schelling). Au bout de 100 millions de tirages, nous n'en avons obtenu aucun. Et nous n'en avons toujours trouvé aucun même après 200 millions de tirages. Les situations les plus probables correspondent aux plus fortes entropies, c'est-à-dire aux échiquiers où la ségrégation est la plus faible. Dans $97 \%$ des échiquiers, un individu au moins n'a aucun voisin semblable et la ségrégation vaut en moyenne 0,15. Ensuite, le cas le plus probable est celui où l'individu le moins satisfait n’a qu'un individu semblable sur six voisins. La ségrégation monte alors à 0,16. Et ainsi de suite.

Le seuil de tolérance de 1/3 retenu par Schelling est donc tout à fait particulier. C'est le seuil à partir duquel le mélange devient quasiment impossible. Pointons dès à présent le problème que cela pose. En négligeant l'étude de la statique (mais il est vrai que Schelling n'avait pas les moyens de mener autant de simulations que nous), Schelling se condamne à manquer d'un point de référence à partir duquel juger un effet ségrégationniste. Il y a ici une forme d'illusion cognitive qui consiste à croire que, avec une intolérance de seulement $1 / 3$, il y aurait encore beaucoup de possibilités de mélanges des blancs et noirs et, donc, que le résultat obtenu par Schelling serait surprenant. Mais, 
précisément, cela n'a rien de surprenant : la statistique nous montre qu'en réalité ces échiquiers peu ségrégués pour ce seuil de tolérance sont inexistants.

\section{Des simulations du jeu de Schelling}

Pour aller plus loin, il faut à présent procéder aux simulations de ce jeu. Cela doit permettre de mesurer deux choses. Tout d'abord, évidemment, la ségrégation finale. Mais également, la tolérance exacte des individus. En effet, la présentation que Schelling donne de son modèle est trompeuse. Il nous présente le seuil de tolérance individuelle comme une variable continue alors qu'il s'agit d'une variable discrète, ce qui bouleverse l'interprétation des résultats obtenus. Prenons le cas où un individu a trois voisins. Comment se traduit concrètement son désir d'avoir plus d'un tiers de voisins semblables à lui-même ? Par le rejet des situations où, sur ses trois voisins, aucun ou un seul lui ressemble et, en réalité, il n'accepte que les situations où deux voisins ou plus sont de sa couleur. Autrement dit, il veut faire partie de la majorité. Il rejette 0/3 ou $1 / 3$ et accepte uniquement $2 / 3$ ou $3 / 3$. On peut toujours dire qu'il est prêt à accepter $34 \%$ de semblables, mais puisque dans ces simulations, la tolérance varie selon des sauts discrets, cela signifie qu'en réalité son niveau minimum d'intolérance est de 2/3. Ce calcul peut bien sûr être fait pour chaque nombre possible de voisins de 1 à 8 . Par exemple, dans le cas de Schelling où le seuil est de plus de 1/3, l'intolérance minimale réelle correspond à : 1 voisin semblable au moins parmi 1 voisin en tout, 1 semblable au moins parmi 2, 2 semblables au moins parmi 3, 2 semblables au moins parmi 4, 2 semblables parmi 5, 3 parmi 6, 3 parmi 7 et, enfin, 3 parmi 8. Les proportions minimales acceptées sont donc les suivantes : 1/1,1/2, 2/3, 2/4, 2/5, 3/6, 3/7 et 3/8 (le numérateur indiquant le nombre minimum de voisins identiques et le dénominateur le nombre total de voisins). Comme on peut le constater sur cette série, le seuil de $34 \%$ choisi par Schelling n'existe dans aucun des cas de nombres de voisins susceptibles de se rencontrer concrètement au cours d'une simulation. On peut ensuite facilement établir cette série de proportions minimales réellement acceptées pour tout seuil d’intolérance fixé dans le continu (entre 0 et 1). Cela permet pour un seuil donné de calculer l'intolérance minimale moyenne qui est effectivement en cause dans le modèle en pondérant chacune de ces proportions par le nombre de fois où elle a été rencontrée au cours d'une simulation, c'est-à-dire en prenant en compte toutes les étapes qui conduisent à un état final d'équilibre. Dans la suite de cette étude, nous appellerons intolérance effective le résultat de ce calcul.

Il faut également observer que les conditions initiales du modèle de Schelling sont tout à fait exceptionnelles : elles sont toujours très proches de l'alternance parfaite - ce qui a pour conséquence de relativiser fortement la généralité du modèle. Ici comme ailleurs il est difficile de tirer des conclusions générales d'un modèle dont les conditions initiales sont si singulières. Bien entendu, dans la mesure où nous discutons du modèle de Schelling, nous avons effectué des simulations en partant exactement des mêmes conditions initiales que lui. À proprement parler, après retrait des pions au 
hasard, il s'agit d'un départ quasi alterné (avec coins vides). Mais nous avons également mené des simulations en partant de conditions initiales de répartition des pièces sur l'échiquier totalement au hasard. Comme nous le verrons, la comparaison des deux est riche d'enseignements.

D’autres particularités des règles du jeu de Schelling peuvent a priori avoir une incidence sur la ségrégation finale, mais comme leur effet est beaucoup plus marginal on peut sans difficulté s'en affranchir (Forsé, Parodi, 2010). Le fait qu'il soit possible de s'en affranchir sans altérer significativement la ségrégation obtenue en suivant scrupuleusement les consignes de Schelling est d'ailleurs à mettre à son crédit puisque cela montre que, relativement à ces quelques contraintes, son modèle est robuste.

Tableau N ${ }^{\circ} 1$ : Résultats des simulations du Jeu de Schelling avec départs quasi alternés OU AU HASARD. SOURCE : SIMULATIONS DES AUTEURS.

\begin{tabular}{|c|c|c|c|c|}
\hline départ & \multicolumn{2}{|c|}{ au hasard } & \multicolumn{2}{|c|}{ quasi alterné } \\
\hline Seuil théorique & $\begin{array}{c}\text { Intolérance } \\
\text { effective }\end{array}$ & Ségrégation & $\begin{array}{c}\text { Intolérance } \\
\text { effective }\end{array}$ & Ségrégation \\
\hline $0(0 \%)$ & $25 \%$ & 0,21 & $23 \%$ & 0,10 \\
\hline $1 / 8(13 \%)$ & $26 \%$ & 0,21 & $24 \%$ & 0,09 \\
\hline $1 / 7(15 \%)$ & $28 \%$ & 0,22 & $26 \%$ & 0,09 \\
\hline 1/6 (17 \%) & $31 \%$ & 0,25 & $29 \%$ & 0,10 \\
\hline $1 / 5(21 \%)$ & $35 \%$ & 0,31 & $33 \%$ & 0,13 \\
\hline $1 / 4(26 \%)$ & $40 \%$ & 0,40 & $39 \%$ & 0,23 \\
\hline $2 / 7(29 \%)$ & $42 \%$ & 0,46 & $41 \%$ & 0,32 \\
\hline $1 / 3(34 \%)$ & $50 \%$ & 0,57 & $50 \%$ & 0,57 \\
\hline $3 / 8(38 \%)$ & $51 \%$ & 0,59 & $50 \%$ & 0,60 \\
\hline $2 / 5(41 \%)$ & $55 \%$ & 0,66 & $54 \%$ & 0,67 \\
\hline $3 / 7(43 \%)$ & $57 \%$ & 0,70 & $56 \%$ & 0,71 \\
\hline $1 / 2(51 \%)$ & $69 \%$ & 0,84 & $69 \%$ & 0,85 \\
\hline $4 / 7(58 \%)$ & $70 \%$ & 0,88 & $70 \%$ & 0,88 \\
\hline
\end{tabular}

Note : Les calculs sont effectués pour chaque seuil sur 1000 simulations pour le départ quasi alterné (avec coins vides) pour reprendre les conditions initiales de Schelling et également sur 1000 simulations pour le départ au hasard. Il y a 45 pions sur chaque échiquier ( 20 noirs, 20 blancs et 5 de couleur aléatoire à chaque départ). Le seuil minimal indique ici, au sens de Schelling, le seuil que les individus se doivent de dépasser strictement. Ce tableau présente par ordre croissant tous les seuils minimaux possibles de 0 à $4 / 7$ (exclus). Entre parenthèses figurent, à côté des seuils théoriques, leur valeur en pourcentages.

Lecture : Pour le seuil théorique de plus de $1 / 3$, soit $34 \%$ au minimum, les individus déménagent en moyenne jusqu'à être avec $50 \%$ de voisins semblables et, pour un départ quasi alterné ou au hasard, la ségrégation monte en moyenne à 0,57. Rappelons que cet indice de ségrégation varie de 0 pour un mélange parfait à 1 pour une ségrégation totale.

Le tableau $\mathrm{n}^{\circ} 1$ présente les résultats des simulations correspondant à différents seuils d'exigence individuelle. Il y a quatre enseignements à en tirer. 
- Tout d'abord, l'intolérance effective est toujours largement supérieure à ce que suggère le seuil théorique présenté par Schelling.

- Dans le cas du seuil de plus d'un tiers retenu par Schelling, cette intolérance est en réalité de l'ordre de 50 \%. Le seuil de Schelling est une pure abstraction et, en réalité, les individus dans ce modèle ne sont satisfaits que dans des voisinages où leur couleur est majoritaire. La ségrégation observée est donc un résultat logique et il n'y a aucune raison de voir ici de paradoxe : si chacun veut faire partie de la majorité, personne n'accepte d'être en minorité et la seule solution consiste alors à créer des ghettos. Ceci est parfaitement cohérent avec notre premier résultat qui montrait que le mélange n'était pas possible à partir de ce seuil théorique de 1/3 : il n'existe pas, en effet, d'échiquier où deux populations sont mélangées alors que chacun veut que sa couleur soit majoritaire dans son voisinage.

- À l'inverse, lorsque l'intolérance effective est d'à peu près $1 / 3$, la ségrégation se situe aux environs de 0,25 pour des départs au hasard (et elle tombe même à 0,13 pour des départs quasi alternés). Or, ce chiffre correspond à ce que l'on peut attendre sous le seul effet du hasard puisque c'est l'ordre de grandeur de la ségrégation atteinte lorsque l'on se contente de jeter au hasard le même nombre de pièces sur un échiquier et que l'on se borne à constater que la distribution obtenue satisfait le seuil d'intolérance individuelle d'un tiers inclus (après avoir répété l'opération des millions de fois).

- Les effets d'une certaine dose d'intolérance ne sont pas disproportionnés au regard de la ségrégation constatée. Si l'on prend pour point de départ la situation la moins biaisée, c'est-à-dire celle où les pions sont répartis au hasard sur l'échiquier, et que l'on porte sur un graphique les données du tableau $\mathrm{n}^{\circ} 1$ (avec en abscisse les niveaux d'intolérance individuelle et en ordonnée les niveaux correspondants de ségrégation atteints), le nuage de points obtenu s'écarte très peu, et sans rupture notable, d'une droite de régression à pente positive (c'est-à-dire que plus l'intolérance augmente, plus la ségrégation augmente - et ce au même rythme). Statistiquement, la relation entre intolérance individuelle et ségrégation globale est très largement linéaire $\left(\mathrm{R}^{2}=0,99\right)$. La linéarité est légèrement plus faible dans le cas des départs quasi alternés $\left(\mathrm{R}^{2}=0,96\right)$, mais cela ne fait que refléter le biais dû à la situation exceptionnelle de départ qui permet de maintenir des taux bas de ségrégation tant que l'intolérance effective reste en dessous de 50 \% (nous y reviendrons un peu plus loin).

La conclusion est dès lors claire. En présentant la règle du tiers dans le continu, on masque une intolérance individuelle en réalité beaucoup plus élevée : environ un demi. Le niveau de ségrégation atteint n'a alors rien d'étonnant compte tenu de cette règle réelle de tri que l'on se fixe. Lorsque cette règle est au contraire effectivement aux alentours du tiers, la ségrégation se situe au niveau de ce que l'on observe sous le seul effet du hasard. Il n’y a donc pas à proprement parler d'« effet Schelling ». 


\section{Les présupposés du modèle de Schelling}

Modéliser, c'est toujours caricaturer le réel pour mettre en lumière un phénomène précis. Il y a toutefois des chausse-trappes à éviter et des précautions à prendre avant d'appliquer à la réalité telle ou telle explication proposée par un modèle.

\subsection{II faut pouvoir distinguer précisément les intentions prêtées aux acteurs}

Dans le modèle de Schelling, les individus ont en moyenne 4,7 voisins et les nombres de voisins les plus fréquents sont 3, 4, 5 ou 6 , ce qui correspond à des seuils d'exigence minimale de $2 / 3,2 / 4,2 / 5$ et $3 / 6$ conduisant, comme nous l'avons vu, à une tolérance moyenne effective de 0,50 . Nous sommes censés croire que les individus sont assez tolérants (à $34 \%$ ) alors qu'en fait la plupart de ceux-ci déménageront tant qu'ils ne feront pas partie de la majorité.

Pour prouver l'effet Schelling, il faudrait construire un modèle avec plus de voisins, de manière à mieux modéliser les intentions des acteurs en discriminant réellement entre les situations où les acteurs sont un peu intolérants des situations où ils sont fortement intolérants. Sinon, on attribue à tort une faible intolérance à des acteurs très intolérants et le fait d'obtenir une forte ségrégation n'est pas paradoxal, mais la conséquence d'un défaut de modélisation.

Il y a plusieurs manières d'augmenter le nombre de voisins dans un modèle « à la Schelling » (Laurie, Jaggi, 2003). Mais le résultat va toujours dans le même sens, à savoir que l'effet « paradoxal » disparaît. On notera au passage que, dans tous les cas, ce que nous avons appelé l'intolérance effective apparaît comme l'information réellement pertinente dans ce type de modèle, et non le seuil théorique de Schelling.

\subsection{II faut toujours prêter attention aux conditions initiales d'un modèle}

Les conditions initiales d'un modèle peuvent introduire des déviations significatives par rapport à l'effet général modélisé. En toute rigueur, il faut partir des conditions initiales les plus neutres possible. Cette idée a été formalisée en théorie des probabilités avec le principe de l'« entropie max » : le point de départ que l'on doit retenir est celui qui a le plus faible contenu en information (Jaynes, 2003). C'est ce que nous avons fait en choisissant un départ au hasard tandis que Schelling a, lui, choisi un point de départ très particulier : la quasi-alternance.

Le tableau $\mathrm{n}^{\circ} 1$ montre des différences entre les simulations selon le point de départ retenu. Pour de faibles intolérances, la ségrégation résultante est en moyenne plus faible lorsque le départ est quasi-alterné. Puis, à partir du fameux seuil d'un tiers, les ségrégations moyennes deviennent similaires quel que soit le point de départ. L'impression que la ségrégation augmenterait brutalement à partir du seuil d'un tiers s'explique alors non par une sorte de "bifurcation catastrophique » mais, au contraire, par la destruction à partir de ce seuil d'une situation d'alternance quasi parfaite. Loin d'une 
« main invisible » qui construit la ségrégation à partir d'un certain seuil, le modèle rend compte de la disparition d'un état initial instable - la quasi-alternance - que Schelling a construit de sa propre main. Avec un tel départ, les règles du jeu de Schelling ne sont même pas nécessaires pour que la ségrégation augmente. Même des mouvements purement aléatoires conduiraient à un accroissement similaire.

La ségrégation finale demeure d'ailleurs très faible et proche de la ségrégation initiale quand le point de départ est quasi-alterné et que le seuil d'intolérance est effectivement bas. En effet, il suffit parfois d'un ou deux déménagements pour satisfaire tout le monde. Le jeu n’a pas le temps de s'écarter réellement du point de départ. On parle alors de dépendance aux conditions initiales, ce qui enfreint le principe ergodique et empêche de tirer du modèle des conclusions générales. Le principe ergodique consiste en effet à négliger les conditions initiales en considérant que la dynamique finit par effacer toute spécificité liée au point de départ : seul compte, dans ces conditions, les caractéristiques communes du problème. Mais ici, ce n'est pas le cas. Il y a une dépendance au chemin (path dependency) et il faut alors tenir compte des spécificités historiques.

\subsection{II faut se demander si ce que le modèle a négligé est négligeable}

Un modèle néglige toujours certains phénomènes, il est donc impératif de se demander si ce qui a été négligé était négligeable quant au résultat mis en avant par le modèle. Une des hypothèses implicites du modèle de Schelling est que les satisfaits ne déménagent jamais. Autrement dit, le processus migratoire n'est pas simplement une marche sélective a minima, qui imposerait seulement d'aller d'une position insatisfaisante à une position satisfaisante par un chemin quelconque, c'est dans le même temps une marche sélective qui impose des mobilités potentiellement différentes entre les pions. Tout quartier totalement homogène a un potentiel nul de voir ses habitants déménager. Les physiciens parlent à ce propos de phénomènes de nucléation (Vinkovic, Kirman, 2006). On notera que ces phénomènes de nucléation sont des phénomènes cinétiques qui expliquent des écarts par rapport à l'équilibre stable. Ce sont des états transitoires ou des états en équilibre métastable (c'est-à-dire des états en équilibre précaire, une petite fluctuation pouvant suffire pour ramener le système à l'équilibre stable). Le modèle de Schelling provoque ainsi un équilibre métastable à l'aide d'une règle dont la portée reste implicite : la règle qui consiste à ne jamais faire déménager des satisfaits. Une telle règle revient, pour utiliser une image, à conseiller à un cuisinier de mélanger une pâte à crêpe sans toucher aux grumeaux et à trouver surprenant que les grumeaux n'aient pas disparu mais aient grossi à l'issue du mélange.

Cette règle implicite explique que la ségrégation finale des échiquiers obtenus par la méthode de Schelling pour un départ au hasard soit un peu supérieure à la ségrégation calculée dans la première partie (qui livrait la statistique correspondant aux propriétés statiques du jeu). Ceci dit, les résultats s'en écartent très peu. 
Le modèle de Schelling néglige donc les déménagements effectués pour d'autres raisons que l'intolérance. Cela semble parfaitement anodin, or cela ne l'est pas pour les conclusions de son modèle. En prenant en considération ces autres déménagements, on observerait spontanément la disparition de quartiers homogènes qui ne sont que des formations transitoires. La conclusion de Schelling est donc très fragile car, au fond, il arrête le film au moment où, par accident, des quartiers homogènes se sont formés. Ce qu'il a négligé dans son modèle n'est ainsi pas négligeable, mais a au contraire son importance pour les conclusions mêmes de son modèle.

\section{Une tendance spontanée au mélange}

La ségrégation urbaine a de nombreuses causes politiques, économiques et sociales. Et il faut considérer ces causes comme structurant l'espace sur la longue durée étant donné la lente évolution du paysage urbain et l'attachement des habitants à leur quartier et à ceux qui y habitent. C’est dire que la ségrégation urbaine est un phénomène qui peut difficilement être expliqué par un unique facteur. Dans le choix d'une famille d'habiter un quartier, le revenu du ménage pèse certainement lourdement, mais également la proximité d'une bonne école pour les enfants ou un temps de trajet raisonnable jusqu'au lieu de travail des parents. Autant de critères de choix auxquels on peut encore ajouter la proximité des grands-parents, la connaissance du quartier, les infrastructures publiques comme les transports en commun, la présence d'une crèche, l'animation ou la réputation du quartier, etc. Et, aussi, le désir éventuel d'un entre-soi socio-économique ou socio-ethnique.

Le modèle de Schelling a remporté un vif succès parce qu'il semblait illustrer parfaitement l'idée que nous n'obtenons pas toujours au niveau collectif ce que chacun vise individuellement. La ségrégation urbaine passait pour un effet non voulu de comportements modérément racistes. Encore récemment, certains chercheurs (Fossett, 2006) soutenaient, en suivant Schelling, qu'aux États-Unis de grands ghettos pouvaient naître d'intentions individuelles faiblement intolérantes tandis que d'autres chercheurs montraient au contraire que les niveaux de ségrégation étaient tels dans ce pays qu'ils ne pouvaient s'expliquer que par des attitudes individuelles fortement discriminatoires, même si elles sont aujourd'hui plus voilées (Massey, Denton, 1993 ; Yinger, 1995). Dans ce débat, le modèle de Schelling paraissait tomber à point nommé pour expliquer qu'il y ait encore de la ségrégation dans des sociétés pourtant généralement de plus en plus tolérantes ou qui se jugent telles. C'est toutefois un peu court et les sciences sociales doivent prendre au sérieux la question de l'application de leurs modèles. Il ne suffit pas qu'un modèle exhibe un effet, il faut encore que le modèle soit pertinent et que l'on n'attribue pas à tort tel modèle à tel phénomène.

Considérons par exemple l'agglomération parisienne. Les travaux d'Edmond Préteceille $(2006$; 2016) permettent de relativiser fortement les discours dramatiques des médias et des politiques sur les évolutions de la ségrégation, qui ne parlent que de ghettos, de fractures, voire d'apartheid. Or, les situations de mixité dominent large- 
ment au sein de l'agglomération, à la fois sur le plan socio-économique et sur le plan ethno-racial, mais ces situations n'attirent pas l'attention du public. Il y a effectivement des zones où la ségrégation augmente un peu, notamment parmi les populations aisées qui recherchent l'entre-soi à Neuilly-sur-Seine ou dans le $16^{\mathrm{e}}$ arrondissement de Paris, mais précisément ce n'est pas la tendance générale. De même, il y a bien des zones fortement ségréguées sur le plan ethno-racial, mais ce sont manifestement des zones d'entrée de l'immigration et, à partir de ces "sas », on observe bien ensuite une diffusion de ces groupes ethno-raciaux dans le reste de l'agglomération. On peut sur ce point citer longuement Préteceille (2016 : 43) :

Quant à la ségrégation ethno-raciale, on a vu qu'elle a diminué dans l'ensemble. Ce qui n'empêche pas, comme pour la ségrégation socio-économique, que des situations locales de forte concentration d'immigrés se soient renforcées : on comptait en 2008 dans l'aire urbaine de Paris 412 IRIS où l'ensemble des immigrés originaires du Maghreb, d'Afrique subsaharienne et d'Asie et les Français nés dans les DOM-TOM constituaient plus de $50 \%$ de la population locale, contre 223 seulement en 1999. Mais si leur ségrégation d'ensemble par rapport aux Français nés en France métropolitaine a baissé, c'est qu'hors de ces quartiers, ces immigrés sont plus mélangés encore avec les Français. Et ceux qui habitent ces quartiers où ils sont majoritaires localement ne sont que $24 \%$ du total du groupe, dont plus des trois quarts résident donc dans des quartiers où ils sont minoritaires. Il est donc, là aussi, parfaitement abusif de laisser entendre que les immigrés résident tous dans des ghettos d'immigrés.

Partant d'un espace structuré, on observe ainsi que la tendance générale est bien celle du mélange, et non l'inverse. Ce résultat est conforme au principe entropique qui dit que, en général, nous produisons plutôt spontanément du désordre, du bruit et de la pollution et, inversement, que l'ordre ou la lutte contre la pollution nous demande un effort spécifique. Le modèle de Schelling nous égare en nous invitant à trouver normal d'inverser cette tendance générale. Il faut au contraire que les sciences sociales ne se satisfassent pas de ce genre d'explication. Lorsqu'un ghetto ou une zone très ségréguée apparaît, il y a de vraies causes, qui doivent faire l'objet d'une enquête empirique pour que l'explication pertinente puisse être trouvée.

\section{Conclusion}

La thèse soutenue par Schelling, selon laquelle on bascule dans une société fortement ségréguée même avec une intolérance individuelle modérée, ne résiste pas à un examen détaillé. Comme nous l'avons vu, lorsque l'intolérance est réellement modérée, la ségrégation est elle-même modérée et lorsque l’intolérance est importante, la ségrégation est évidemment importante - le lien entre ces deux variables étant quasi linéaire. L'étude des contraintes structurelles confirme à cet égard que les ségrégations résultant du jeu de Schelling n'ont rien d'exceptionnel. On ne peut pas dire que le point de basculement vers des ségrégations fortes se rencontre à des niveaux d'intolérance plus faibles que prévus. Si certains ont été persuadés qu'il y avait davantage dans ce modèle 
que les résultats triviaux auxquels il aboutit, cela tient finalement à ce que deux tautologies s'y télescopent pour conduire, si l'on n'y prend garde, à un paralogisme : oui, de petites exigences sur son voisinage créent un peu d'ordre ; oui, si tous souhaitent être majoritaires alors il n'y a pas de minorités possibles et la ségrégation est élevée ; mais non, la ségrégation n'est pas en décalage par rapport à ce que l'on peut attendre compte tenu des lois du hasard et de ce que chacun souhaite effectivement. Le modèle de Schelling ne permet pas de conclure que de grands ghettos naissent d'innocentes décisions individuelles.

Au-delà de ce modèle, il y a encore une autre leçon à tirer, à savoir que la force illustrative d'un modèle ne doit pas nous leurrer. C'est pourquoi il faut le soumettre à l'épreuve du réel et mettre au jour tous ses a priori en posant les bonnes questions. Ainsi, quand on parle d'un effet social, il y a forcément un point de référence à partir duquel juger l'effet (tout comme en théorie de la relativité, le mouvement n'existe qu'une fois posé un point fixe). Première question donc : quel est le point de référence du modèle ? Quand on modélise une intention, celle-ci ne doit pas être ambivalente, au risque sinon d'une surinterprétation. Il faut donc se demander en deuxième lieu si cette ambivalence est bien absente. Si l'on veut conclure à un effet général, les conclusions ne doivent pas dépendre du choix des conditions initiales. D'où une troisième question : le point de départ du modèle est-il vraiment neutre ? Enfin, quatrième nécessaire vérification, s'il est normal qu'un modèle néglige des phénomènes, en retour il faut tout de même s'assurer que ce qui a été négligé ne perturbe pas fortement les conclusions du modèle. Sinon, on finit par ne voir que ce que l'on voulait voir.

\section{Bibliographie}

Forsé M., Parodi M. (2010), « Low levels of ethnic intolerance do not create large ghettos, A discussion about an interpretation of Schelling's model ", L'Année sociologique, vol. $60, n^{\circ} 2$, p. $445-473$.

Fossett M. (2006), « Ethnic Preferences, Social Distance Dynamics, and Residential

Segregation: Theoretical Explorations Using Simulation Analysis », Journal of Mathematical Sociology, vol. 30, p. 185-274.

Jaynes E. T. (2003), Probability Theory. The Logic of Science, Cambridge, Cambridge University Press.

Kapur J. N., Kesavan H. K. (1992), Entropy Maximisation Principles with Applications, San Diego, Academic Press.

Laurie A. J., Jaggi N. K. (2003), « Role of 'Vision' in Neighbourhood Racial Segregation: A Variant of the Schelling Segregation Model », Urban Studies, vol. 40, n 13, p. 26872704.

Massey D. S., Denton N. A. (1993), American Apartheid: Segregation and the Making of the Underclass, Cambridge, Harvard University Press. 
Préteceille E. (2006), « La ségrégation sociale a-t-elle augmenté? La métropole parisienne entre polarisation et mixité », Sociétés contemporaines, vol. 62, n 2, p. 69-93.

Préteceille E. (2016), « La métropole parisienne à la croisée des chemins : inégalités et ségrégations, traiter les effets ou s'attaquer aux causes », Paris. Les cahiers de la métropole, p. 36-45.

Ruoff G., Schneider G. (2006), « Segregation in the Classroom: An Empirical test of the Schelling Model », Rationality and Society, vol. 18, n 1, p. 95-117.

Schelling T. C. (1971), « Dynamic Models of Segregation », Journal of Mathematical Sociology, vol. 1, n² 2, p. 143-186.

Schelling T. C. (1978), Micromotives and Macrobehavior, New York, Norton.

Shannon C. E. (1948), " A Mathematical Theory of Communication », Bell System Technical Journal, vol. 27, p. 379-423 et p. 623-656.

Vinkovic D., Kirman A. (2006), "A Physical Analogue of the Schelling Model », Proceedings of the National Academy of Sciences, vol. 103, n 51, p. 19261-19265.

Yinger J. (1995), Closed Doors, Opportunities Lost: The Continuing Costs of Housing Discrimination, New York, Russell Sage Foundation. 